\title{
Welwitschin minabilis.
}

Die Gardener's chronicle vom 30. October d. J., pag. 565 (der Zeitschrift "Nature" entnommen), führt an, dass von Mr. Bower im Jodrell-Laboratorium in Kew, Keimungsversuche mit WelwitschiaSamen vorgenommen wurden, durch welche sich das interessante Factum feststellt, dass die beiden colossalen Blätter derselben, welche vormals für bleibende Cotyledone gehalten wurden, es nicht sind, sondern in der That die ersten und einzigen Stammblätter, die an der Pflanze sofort verbleiben. Die eigentlichen Cotyledone befinden sich zuerst für einige Zeit innerhalb des Samens, werden aber zuletat ganz fiei, ungeachtet dessen ist dennoch eine Verbindung mit dem Endospermium durch einen seitlichen Fortsatz vom Tigellum oder jenem Theil des Stammes unterhalb der Cotyledone hergestellt, der ähnlich einer Wurzel in das Endospermium vorragt und gleichsam eine entfernte Analogie mit dem Fusse einiger Kryptogamen andeutet.

F. Antoine.

\section{Iiteraturberichte.}

Ilandbuch der Botanik, heransgegeben von Prof. Dr. A. Schenk, unter Mitwirkung von Prof. Cohn in Breslau, Dr. Detmer in Jena, Dr. O. Drude in Dresden, Prof. Frank in Leipzig und Anderen. Breslau. E. Trewendt. $1879-1880$.

Dieses auf drei Bände berechnete Handbuch der Botanik, von denen der erste nunmehr vollendet vorliegt, erscheint in der wahrhaft grossartig angelegten „Encyklopädie der Naturwissenschaften“, deren Redaction von dem unternehmenden Verleger in die Hände von Rich. Fleischer gelegt wurde. Der vorliegende, 750 Grossoctavseiten umschliessende, von zahlreichen Holzschnitten gezierte, reich ausgestattete Band weicht von ähnlichen Werken in der ganzen Anlage ab, inderm er ans in sich abgeschlossenen Abhandlungen besteht, in welchen - nur zum Vortheile des Lesers - die Eigenart des Autors zur vollen Geltung kommt. Auch in der Reihenfolge der Abhandlungen gibt sich eine grosse Freiheit zu erkennen, wie aus dem Inhalte des ersten Bandes zu ersehen ist, welcher sich folgendermassen gliedert: 1. Wechselbeziehungen zwischen den Blumen und den ihre Kreuzung vermittelnden Insecten, von Dr. Hermann Müller. 2. Die insectenfressenden Pflanzen, von Dr. O. Drude. 3. Die Gefässkryptogamen, von Prof. Sadebeck. 4. Die Pflanzenkrankheiten, von Prof. Frank, endlich 5. Die Morphologie der Phanerogamen, von Dr. 0. Drude. Auf den ersten Blick erseheint diese Anordnung allerdings als ein Chaos, allein bei dem Plane, den Gegenstand in getrennten Essays zu behandeln, ist die Aufeinanderfolge derselben ziemlich gleichgiltig, auch beim Gebrauche kaum störend, und bietet den Vortheil, das Werk verhältnissmässig rasch erscheinen lạssen zu können, indem fast jede Abhandlung, so wie sie fertig ist, sofort dem 
Werke einverleibt werden kann. Das Buch wird wohl jedem Botaniker zur Hand sein müssen, ähnlich wie das ältere Hofmeister'sche Werk, da die einzelnen Abhandlungen nicht nur mit grossei Ausführlichkeit durchgeführt sind, sondern auch zahlreiche neue Beobachtungen bringen. Einzelne der Abhandlungen, wie die erstgenannte, ist eigentlich eine grosse Originaluntersuchung über die Beziehung zwischen Blmmen und Insecten und enthält einen sehr vollständigen Literaturnachweis. Ein Gleiches gilt für die von Frank herrührende Abhandlung über die Pflanzenkrankheiten, welche besonders deshalb so wichtig erscheint, als sie den Gegenstand ohne jede Einseitigkeit behandelt, indem darin nicht nur auf die in neuerer Zeit vielfach wissenschaftlich bearbeitoten Pilzkrankheiten, sondern auf alle anderen bisher bekannt gewordenen pathologischen Erscheinungen im Pflanzenreiche in ebenmässiger Weise Rücksicht genommen wird. In wahrhaft meisterhafter Weise ist 0 . Drude seinem Thema gerecht geworden, indem er in die erdrückende Masse des Materiales Ordnung gebracht und den Gegenstand mit einer Klarheit vorgetragen hat, so dass sein zum Handbuch gelieferter Beitrag zu den besten gezählt werden muss, was die Literatur Zusammenfassendes über die Morphologie der Phanerogamen besitzt. Dabei fehlt es nicht an origineller Auffassung in wesentlichen Punkten. Auch die anderen hier nicht besonders hervorgehobenen Theile des Handbuches sind in musterhafter Weise durchgeführt, wie die Namen der Autoren auch nur erwarten liessen.

Bei dem allgemeinen Interesse, welches die "Encyklopädie der Naturwissenschaften" jedem Naturforscher, welcher Richtung er auch immer angehöre, bietet, erscheint es vielleicht manchem Leser erwünscht, den Plan des ganzen Werkes kennen zu lernen. Ausser Botanik wird die Encyklopädie noch folgende Disciplinen behandeln: Zoologie und Anthropologie (v. Hellwald und G. Jäger), Mathematik (Sehlömilch), Mineralogie (Kenngott), Physik (Zech), Chemie (Ladenburg), Astronomie (v. Oppolzer), Pharmakognosie (Wittstein). Die Namen der Redacteure bürgen wohl genügend für die gediegene Durchführung der einzelnen Werke. Bis jetzt sind ausser dem angezeigten Bande der Botanik noch die ersten Bände der Zoologie und Mathematik erschienen.

J. W.

Die Krankheiten der Pflanzen. Ein Handbuch etc. von Dr. B. Frank, Prof. an der Universität Leipzig. Erste Hälfte. Mit 62 Holzschnitten. Breslau, Trewendt 1880. 400 Seiten $8^{0}$.

Wir begrüssen dieses Werk mit aufrichtiger Freude; denn wenn in der wahrhaft grossen botanischen Literatur sich eine Lücke fühlbar machte, so war es der Mangel an einem dem heutigen Standpunkte der Forschung entsprechenden grösseren und allseitigen Werke über die Pathologie der Gewächse. Grössere Monographien über Pflanzenkrankheiten liegen wohl vor, darunter geradezu ausgezeichnete Abhandlungen und Werke, und namentlich sind die Pilzkrankheiten der Cuiturgewächse in einer dem Bedürfnisse entsprechenden Weise in neuerer Zeit bearbeitet worden. Man wolle sich in dieser Beziehung 
nur der Namen J. Kühn, R. Hartig und Soraner erinnern. Aber wenn es sich um andere als mykologisch-pathologische Fragen handelte, namentlich um krankhafte Erscheinungen wildwachsender Pflanzen, so ist man ganz und gar auf ältere Schriften angewiesen, wie auf die bekannten Werke von Meyer (1841), Moquin-Tandon (1841) und Wigand (1850). So werthvoll nun diese Schriften für ihre Zeit waren, für uns sind sie bis auf einzelne Capitel und gewisse. Einzelnbeobachtungen heute kaum mehr brauchbar, da ja jene Disciplinen, auf welche sich Teratologie und Pathologie, wenn selbe eine wissenschaftliche Begründung erfahren wollen, stützen müssen, namentlich die Physiologie, in den letzten drei Decennien grosse Fortschritte aufzuweisen haben, so dass viele pathologische Fragen nunmehr erst lösbar geworden sind. Der bekannte und geschätzte Autor, dem wir sowohl auf anatomischem wie physiologischem Gebiete sehr werthvolle Untersuchungen verdanken, hat seine Aufgabe in durchaus trefflicher Weise gelöst, indem er nicht nur die ältere und neuere pathologische Literatur sehr sorgfältig benützte und selbst eine grosse Zahl neuer Beobachtungen bringt, sondern alle Partien des ausgedehnten und verwickelten Gegenstandes lichtvoll und dem heutigen Stande der Grunddisciplinen völlig entsprechend gelöst hat. Um nur eine beiläufige Vorstellung von der Art und Weise zu geben, wie der Autor seinen Gegenstand behandelt, sei hier die Gliederung des den Wunden gewidmeten Capitels gegeben: Allgemeines. A. Die unmittelbaren Folgen der Verwundung für das Leben. I. Abgeschnittene Pflanzentheile (Welken abgeschnittener Sprosse etc.). II. Folgen unpassender Veredlung: III. Verstümmlung von Samen. IV. Verlust und Verletzung von Wurzeln. V. Verstümmlung des Stammes und der Zweige. VI. Verlust der Laubblätter. VII. Rinden- und Holzverletzung (Insectenschäden etc.). VIII. Verletzung von Blättern, Blüthen und Früchten. IX. Abnorme Secretion als Begleiterscheinung der Wunden. B. Wundenheilung. I. Heilung durch Wundkork. II. H. durch Callus. III. H. durch Ueberwallung (Maserbildung; Stammverwachsung etc.). C. Zersetzungserscheinungen des Holzes. D. Behandlung der Wunden. Die bis jetzt erschienene erste Hälfte des Buches enthält folgende Abschnitte: 1. Einleitung. 2. Der Iebende und todte Zustand der Pflanzenzelle. 3. Wunden. 4. Krankheiten, welche durch Einflüsse der unorganischen Natur hervorgebracht werden. 5. Durch andere Pfianzen verursachte Krankheiten. Der letzgenannte Abschnitt wird in der noch im Laufe dieses Jahres in Anssicht gestellten Schlusslieferung zum Abschluss gebracht werden.

J. W.

Pinguicula alpina, als insectenfressende Pflanze und in anatomischer Beziehung. Von Prof. Jul. Klein, Sonderabdrnck aus Cohu's Beiträgen zur Biologie der Pflanzen. III. Bd., 2. Heft (mit 2 Tafeln.)

Der Verf. hatte Gelegenheit, im Sommer 1878 die bei dem steyrischen Bade Neuhaus reichlich vorkommende Pinguicula alpina näher zu untersuchen. Die Resultate seiner. Beobachtungen sind in der vorliegenden Arbeit niedergelegt. Der Verf. fand zwei verschie- 
dene Formen der Pinguicula alpina, die eine mit gelblich-grünen Blättern, die andere mit rothbraunen Blättern; er vermuthet in beiden Formen nur Standorts-Varietäten vor sich zu haben. Im ersten Capitel entscheidet Verf. die Frage, ob Pinguicula alpina eine insectenfressende Pflanze ist oder nicht. Zu diesem Behufe wurden Versuche mit kleinen Insecten, mit robem Fleisch, hartgekochtem Eiweiss, mit Stückchen einer Peziza und mit trockenen Semmelbröseln angestellt. Alle genannten Gegenstände bewirkten sowie bei der von Darwin untersuchten $P$. vulgaris auch hier ein Finbiegen der Blattränder, eine stärkere Absonderung der Drüsen; auch konnte eine mehr weniger starke Veränderung dieser Gegenstände in dem stets saner reagirenden Secrete, sowie in der Folge eine theilweise oder ganze Auflösung derselben und in einigen Fällen auch eine Aufsaugung beobachtet werden. Die $P$. alpina kann mithin als insecten- oder fleischfressende und zum Theil auch als pflanzenfressende Pflanze angesehen werden. In Folgendem wird $P$. alpina in anatomischer Beziehung näher betrachtet. Was die Wurzeln botrifft, gibt Verf. an, dass sie sich nicht verzweigen, nichtsdestoweniger aber ein Pericambium besitzen, ähnlich wie es von Fraustadt auch für Dionaea nachgewiesen wurde. Die Zellen der Strangscheide führen doppelt gewellte, radiale Längswände, sie sind das erste Gebilde, das sich aus dem Urmeristem der Wurzelspitze differenzirt. Der anatomische Bau der Wurzeln entspricht, von einem kurzen Theil an deren Basis abgesehen, einem unentwickelten, gleichsam jugendlichen Zustande, da die Tracheengruppen meist nur aus 2-5 Tracheen bestehen und sich gegenseitig nicht berübren. Das kurze unterirdische Stämmohen ist durch den Besitz sehr kurzgliedriger Gefässe ausgezeichnet, deren Querwände durch eine einzige kreisförmige Oeffnung durchbrochen sind. Die Gefässbündel der Wurzel entspringen theils aus dem Gefässkreis des Stämmchens, theils aus der Blattspur. Die Epidermiszellen der Blätter sind chlorophylllos, führen bei den grünblättrigen Formen einen farblosen, bei den rothblättrigen einen röthlichen Zellsaft, ausserdem je einen Zellkern, in dem Krystalloide sich vorfinden. Auf den beiden Blattflächen kommen zahlreiche Spaltöffnungen vor, deren EntwickIung und Bau nichts Bemerkenswerthes darbietet. Die Epidermis der Blattoberseite entwickelte zweierlei Drüsen, gestielte und ungestielte, die der Unterseite nur ungestielte, die übrigens schwach entwickelt sind und kaum über die Epidermis hervorragen. Aus ihrem Auftreten schliesst der Verf., dass die Pinguicula-Arten einst nur einerlei, ungestielte Drusen besassen, aus denen sich später auf der Oberseite die gestielten Drusen entwickelten, womit sich anch die Fähigkeit zum Insectenfang und deren Verdauung ansbildete. Die Tracheen der Blätter sowie der übrigen Theile führen $\mathrm{n}$ i e Luft, sondern stets eine wässrige Flüssigkeit oder einen gelblichbraunen, harzig aussehenden Stoff. Die Mesophyllzellen enthalten reichlich Chlorophyllizorner, die stets Stärkeeinschlüsse besitzen; ausserdem erscheint viel Stärke im Stämmchen und den Wurzeln ruhender Pflanzen. Die Mesophyllzellen bilden wnter sich 
ziemlich grosse, mit Luft erfüllte Intercellularräume. Sehliesslich werden noch einige Beobachtungen an Blüthen mitgetheilt, von denen jene über das Vorkommen gestielter als ungestielter Drusen an sämmtlichen Blüthentheilen hervorzuheben sind.

-h.

Revision von Sargassam und das sogenannte Surgasso-Meer. Von Dr. Otto Kuntze. (Separat-Abdruck aus Engler's botanischen Jahrbüchern. I. Band. 2. Heft.) $8^{\circ}$. 49 S. mit 1 Phototypie und 1 Karte. Leipzig, Wilh. Engelmann. 1880.

Als Einleitung gibt der Verfasser eine kritische Vebersicht über die wichtigere, Sargassum behandeInde Literatur. Er widerlegt ferner die Existenz des Sargassum baciferum als besondere Species durch zahlreiche Gründe (S. 199). Ein weiterer Abschnitt ist der Variabilität der Sargassen nach dem Standorte und in Bezug auf die einzelnen Organe gewidmet (S. 202-212). Hieran schliesst sich eine Vebersicht der Fucaceen-Gattungen, sowie eine Gruppirung der Sargassenformen nach 11 Formenkreisen (Subgregiformen), welche folgende Namen fübren: Sargassum confervoides, S. taeniatum, S. pterocaulon, $S$. medium, S. vulgare, S. ilicifolium, S. hemiphyllum, S. Acinaria, S. obtusatum, S. Horneri, S. scaberioides (S. 213-219). In diese 11 Formenkreise werden die 300 bis jetzt beschriebenen Arten von Sargassum eingereiht und gibt der Verfasser ein vollständiges Synonymen-Register der genannten Gattung (S. 220-230). Den Schluss der vorliegenden Abhandlung bilden Mittheilungen übor die physikalisch-geographische Beschaffenheit des Sargasso-Meeres. Im Gegensatze zu den herrschenden Ansichten gelangt Kuntze zn dem Resultate, ,dass man von einem constanten und bestimmten Aroale des Sargasso-Meeres, welches nur vom Strande abgerissene, absterbende und allmälig untersinkende Fragmente von Sargassum enthalte, nicht reden durfe" (S. 236). Die beigegebene Tafel enthält Phototypien von 26 Sargassum-Formen, leider bedeutend verkleinert; die Karte gibt eine Uebersicht über die verschiedenen Angaben bezüglich des Sargasso-Meeres. Kuntze's Abhandlung beruht anf fleissigen Detailforschungen und liefert beachtenswerthe Angaben für weitere Studien über die vielfach interessanten Sargassen. $\mathrm{R}$.

Ameriean Jemrnal of Serence. Editors Dona and Silliman, 3. Sur. XX. Nr. 115-180. New Hawen 1880. $8^{\circ}$. 332 S. und 4 TafeIn.

Die vorliegenden vier Hefte der genannten Zeitschrift entbalten keine grösseren Abhandlungen botanischen Inhaltes; sie bringen aber. kleinere Mittheilungen, sowie kurze Referate über folgende Publicationen: Action of light on Vegetation (S. 74-76). - Mucorini announced as the chief source of Mineral Coal by Reinsch (S. 150). - De Candolle's Phytography (S. 150-157 und 241-250). - The Ferns of North America by Eaton (S. 157). - Index perfectus ad Caroli Linnaei Species plantarum collatore Ferd. de Müller (S. 157). - Catalogue of North American Musei arranged by Ran and Hervey (S. 157). - Botanical Exploration of West India Islands (S. 158). - The Native Flowers and Ferns of the United States by Thom. 
Meeham (S. 336). - Botany for: High Schools and Colleges by Bessey (S. 337). - Manual of Swedish Pomology by 0. Enenth (S. 337). R. Atti dell' Istituto Veneto di scienze, lett. ed arti Tom. 6 Ser. 5. Vene. zia 1880. Aggiunte alla Flora Trevigiana, per cura del Sc. P. A. Saccardo e G. Bizzozero. Nachträge zur Flora von Treviso von P. A. Saccardo und G. Bizzozero.

Die vorliegende Arbeit ist eine Frgänzung des von Saccardo im Jahre 1863 zu Padua veröffentlichten Prospetto della Flora Trevigiano fanerogama (Uebersicht der Phanerogamenflora der Provinz Treviso). Daselbst wurden 1387 im genannten Florenbezirke vorkommende Arten von wildwachsenden oder eingebürgerten Phanerogamen anfgezählt. In Visiani's Catalogo delle piante vascolari del Veneto (Jahrgang 1869 der Atti dell' Istit. Veneto) erreicht die Anzahl der auf die Provinz Treviso entfallenden Phanerogamen-Arten bereits 1556. Die gegenwärtigen Nachträge bringen einen Zuwachs von 155 Species. Unter den von Saccardo als neu für dio Flora von Treviso aufgeführten Pflanzen befinden sich: Thalictrum elatum, Aquilegia pyrenaica, Delphiniwm montanum DC. (elatum L.), Viola pinnata, Silene linicola, Medicago Pironae, Trifolium pallescens, Saxifraga controversa Stbg., Galium parisiense, Hieracium amplexicaule, Phyteuma Sieberi, Halleri, Campanula carnica, Lithospermum graminifolium, Orobanche Hederae, 0 . ramosa und congesta Rb., Primula spectabilis, Euphorbia Presslii Guss., Ophrys integra (Sacc. Nuovo giorn. botan. Ital. Vol. III. p. 163), O. Bertolonii, Iris foetidissima, Bromus rigidus.

M. Přichoda.

Borbás Dr. Vince: Iráz puszta növényzete (Vegetation der Puszta Iráz) Separat-Abdruck aus ... maquar orvosok és természet vizsgálók XX. nagygyülésének munkálatai (Arbeiten der XX. Versammlang der ungar. Aerzte und Naturforscher). 9 S. $4^{0}$.

Der Verf. schildert in grossen Zügen die Vegetation dieses im westlichen Theile des Biharer Comitates gelegenen Territoriums und zählt dann die von ihm daselbst beobachteten Pflanzen auf. Neu sind Verbascum nigrum L. var. glabrum et perramosum, während die Menthen mit besonderer Vorliebe erörtert werden. Die Arbeit ist jedenfalls ein werthvoller Beitrag zur Kenntniss des Biharer Comitates.

Thämen F. de: Fungi aliqunot novi in terra Kirghisorum a Juliano S e hell.

Sep-Abdruck aus Nuovo Giornale Botanico Italiano 1880. p. 196-199, $8^{\circ}$.

Neu sind: Aecidium Ligulariae, Nonneae ot Kirghisica, Puccinia Schelliana, Uredo Sonchina, Ramularia Menthae, Fusarium parasiticum, Septoria Ephedrae, Epigeios, Schelliana, Nolitangeris et Lemnanthemi und Ascochyta Schelliana. K.

Förster J. B., Beiträge zur Mooshora von Niederösterreich und Westungarn. Separat-Abdruck aus den Verhandlungen der k. k. zoolog.-botanischen Gesellschait in Wien (Jabrgang 1880). is S. $8^{\circ}$.

Die Besprechung der vorliegenden Arbeit erinnert uns unwillkürlich an Jacob Juratzka und dessen Moosflora Oesterreichs, die 
ein Torso geblieben, an dessen Veröffentlichung sich Niemand heranzuwagen scheint. Die somit entstandene Lücke wenigstens theilweise zu überbrücken, sind in erster Reibe dessen Sehülor berufen. Zu diesen gehört anch der Verfasser der vorliegenden Schrift. Derselbe war ein trener Begleiter Juratzka's auf dessen Excursionen in den letzten Lebensjahren, und verleiht schon dieser Umstand allein den Angaben desselben einen hohen Grad von Glaubwürdigkeit. Unter den 304 Laubmoos-Arten sind 10 neu für Niederösterreich, 2 für Ungarn (Pressburger und Eisenburger Comitat) und 1 für OesterreichUngarn. Den Schluss dieser werthvollen Arbeit bildet noch eine Aufzählung von 47 Iuebermoos-Arten. Hoffen wir den Verfasser auf diesem Gebiete noch öfters begrüssen zu können!

$K$.

Verhandlungen und Mittheilungen des siehenbürgischen Vereines für Naturwissensehaften in Hermannstadt. XXX. Jahrg, 1880, LXXXI und 188 Seiten $8^{\mathrm{B}}$ mit 1 Tafel.

Enthält einen Excursionsbericht mit botanischen Angaben über Reusiora, Dealu plaiu und Talmatsch. K.

\section{Correspondenz.}

Königgrätz, den 20. Oetober 1880.

Im Monate Juni besuchte ich wiederholt eine binter NeuKöniggrätz liegende Sumpfwiese und fand daselbst Carex pulicaris L., C. paradoxa Willd, und C. Davalliana Smith so massenhaft verbreitet, dass ein grosser Theil der Wiese von ihnen allein bewachsen war. Hier und an den angrenzenden, bis gegen Roudnička sich ausdehnenden Wiesen kommen noch Drosera rotundifolia, Cirsium rivulare, Salix repens b. rosmarinifolia, Juncus fuscoater, fliformis, Arnica montana, Scorzonera humilis, Carex Pseudocyperus, elongata, flava a. macrorrhyncha, echinata, Pedicularis palustris n. a. vor. Einige von diesen Pflanzen, so namentlich Drosera rotundifolia und Carex pulicaria fand ich, jedoch nur spärlich verbreitet, auch noch im nahen Erlenwalde, der schon mit den ausgedehnten Neu-Königgrätzer Waldungen zusammenhängt. Da vor Jahren, wie mir gesagt wurde, diese Wiese noch bewaldet war und ohne Zweifel floristisch dem naheliegenden Erlenwalde glich, so hätte sich die ursprüngliche Flora erst unlängst zum Theil verändert, indem einige aus dem höherliegenden Walde vor Jahren niedergeschwemmte und seit dieser Zeit hier angesiedelte Pflanzenarten von anderen durch ausserordentlich günstige Lage bevorzugten Pflanzen fast schon verdrängt wurden. Aehnliche Verhältnisse habe ich auch auf einigen sumpfigen, mitten in Nadelholzwäldern liegenden Wiesen auf dem Zuiéńn bei Tremešná in der Nähe 